\title{
Multidisciplinary biopsychosocial rehabilitation for chronic low back pain: the need to present minimal important differences units in meta-analyses
}

\author{
Silvia Gianola ${ }^{1,2^{*}}$ (D) Anita Andreano ${ }^{2}$, Greta Castellini ${ }^{1,3}$, Lorenzo Moja ${ }^{1,3}$ and Maria Grazia Valsecchi
}

\begin{abstract}
Background: The results of meta-analyses are all too often elusive, making it difficult to interpret their relevance for clinical practice. Reporting them in minimal important difference (MID) units could improve the interpretation of evidence in meta-analyses. The aim of this study was to compare, via calculation of MID units, outcomes after multidisciplinary biopsychosocial rehabilitation (MBR) versus usual care for pain relief in chronic low back pain (LBP).

Methods: We re-analyzed the data of a published Cochrane review on MBR. To attribute a MID to each pain instrument, we first searched the literature for MIDs. The MID was imputed for instruments without an established MID. We compared outcomes after MBR versus usual care for chronic LBP in the short ( $<3$ months), mid ( $>3$ and $<12$ months), and long ( $\geq 12$ months) term. The results of the meta-analyses are reported in MID units and interpreted as follows: if the overall effect size was greater than 1, many patients gained clinically important benefits, if it lay between 0.5 and 1.0, an appreciable number benefited, and if it fell below 0.5 few did.

Results: Improvement in back pain was observed in an appreciable number of patients in the short- and mediumterm after MBR: the MID was lower but still close to 1 ( 0.75 and 0.86 MID units, respectively). MBR probably had little or no benefit for the majority of patients in the long-term, where the MID approached 0 (0.27 MID units, confidence interval 0.07-0.48).

Conclusions: Meta-analyses expressed in MID units may offer better insight into the clinical relevance of MBR: the intervention is highly recommended for reducing pain in the short- and medium-term but cannot be recommended for long-term pain reduction since the benefit decays rapidly.
\end{abstract}

Keywords: Rehabilitation, Meta-analysis, Responsiveness, Low back pain, Patient outcome assessment

\section{Background}

Mechanical low back pain (LBP) is the musculoskeletal disorder with the highest prevalence in the adult population [1], and it carries considerable disability and costs for society [2]. The chronic progression of LBP is often considered a biopsychosocial problem characterised by a combination of physical, psychological and social dysfunctions [3] that are typically patient-reported and have

\footnotetext{
* Correspondence: silvia.gianola@grupposandonato.it

${ }^{1}$ Clinical Epidemiology Unit, I.R.C.C.S. Orthopedic Institute Galeazzi, via R

Galeazzi 4, Milan, Italy

${ }^{2}$ Center of Biostatistics for Clinical Epidemiology, School of Medicine and

Surgery, University of Milano-Bicocca, Monza, Italy

Full list of author information is available at the end of the article
}

a subjective nature. There are many different therapeutic interventions for chronic LBP, but none of them is universally accepted as the magic bullet [4]. A recent review [5] reported that non-pharmacological therapies are more common for the treatment of chronic LBP than for acute LBP [6]. One of the most frequently proposed treatments is multidisciplinary biopsychosocial rehabilitation (MBR). MBR is based on the biopsychosocial model, where health and illness are determined by a dynamic interaction between biological (genetic and biochemical), psychological (mood, personality, and behavior), and social factors (cultural, familial, socioeconomic, and medical assistance) [7].

(c) The Author(s). 2018 Open Access This article is distributed under the terms of the Creative Commons Attribution 4.0 International License (http://creativecommons.org/licenses/by/4.0/), which permits unrestricted use, distribution, and reproduction in any medium, provided you give appropriate credit to the original author(s) and the source, provide a link to the Creative Commons license, and indicate if changes were made. The Creative Commons Public Domain Dedication waiver (http://creativecommons.org/publicdomain/zero/1.0/) applies to the data made available in this article, unless otherwise stated. 
Neuroimaging studies have shown that brain regions activated by nociceptive stimuli can also be affected by emotional and behavioral states [8]. Chronic LBP involves central sensitization, a neuropathic pain component, and may induce maladaptive coping strategies and depression [9] in which the effect of the pain becomes more complex, being both a health and a social problem that requires comprehensive care through a multidisciplinary health care team [5]. In this context, the objective of MBR is to improve physical function and modify beliefs and attitudes by addressing psychological issues or targeting social and work-related behaviour.

Meta-analysis (MA) of randomized controlled trials (RCTs) is considered the best approach to identify the actual benefit of a health intervention. A plethora of different instruments are employed to measure rehabilitation outcomes, often using continuous scales, according to the preference of the researchers who designed the study protocol [10-12]. When studies assess the same outcome but measure it differently, the summary statistic usually adopted for meta-analyses is the standardized mean difference (SMD). This metric is obtained for each study by dividing the mean differences between the intervention and the control group by the pooled standard deviation of the outcome [13]. This approach has two drawbacks: first, the effect of the same magnitude will appear different if the study populations are heterogeneous [13]; second, the effect size expressed in standard deviation units is difficult for most health professionals to interpret [14]. In addition, due to the subjective nature of the outcome variables, the cumulative estimate of the treatment effect needs to be presented as a clinically relevant measure in order to illustrate the benefit of the intervention to patients.

To overcome these limitations, the minimal important difference (MID) can be adopted as the summary statistic. The MID is defined as "the smallest difference in score in the outcome of interest that informed patients or informed proxies perceive as important, either beneficial or harmful, and which would lead the patient or clinician to consider a change in the management." [15] Reporting study results in MID units instead of standard deviation (SD) units for individual studies, and consequently for the pooled effect, can provide a uniform metric, bypassing the issues related to the use of SMD and facilitate the interpretation of results [16].

\section{Aim}

Our aim was to compare via meta-analyses in MID units the effects of MBR versus usual care. To do this, we reanalysed the data from a Cochrane review on "Multidisciplinary biopsychosocial rehabilitation for chronic LBP" [17]; the results are reported in MID units and the implications of this approach versus the traditional one are discussed. We selected this review as a case study because it addresses a relevant health problem in rehabilitation and because Cochrane reviews use a standardised methodology, making them an optimal source for informed choice in health care [18].

\section{Methods}

\section{Case study meta-analysis}

Using the data from the Cochrane review by Kamper and colleagues [17], we analyzed the summary effects for the following comparison: "MBR versus usual care in chronic LBP populations" in the short ( $<3$ months), medium ( $>$ 3 months and $<12$ months), and long ( $\geq 12$ months) term. MBR was defined as an intervention involving a physical component (e.g., an exercise program) and at least one other element from the biopsychosocial model that is psychological, social or occupational (e.g., educational psychological counselling) [17]. Usual care consisted of routine treatments: medication prescription (e.g., analgesics) [19-25]; any type of treatment, including routine physiotherapy and/or alternative medicine and/or pain medications [26-30]; medical care as directed by a medical specialist [31-33].

We focused on perceived pain, the most common patient-reported outcome in low back pain rehabilitation [10], which is variously measured across studies, with some using the visual analogue scale (VAS), the numerical rating scale (NRS), or the Short Form 36 (SF-36) Body Pain Index, and others not reporting the instrument.

We updated the case study meta-analysis and ran the original search strategy [34] in the MEDLINE database from February 2014 until December 2017.

\section{Literature search for anchor-based MID values and imputation of missing MID}

Anchor-based and distribution based methods can be employed to establish a MID for an outcome measure in a defined population, however, there is no consensus on which technique is most appropriate $[35,36]$. We chose anchor-based methods because they rely on an external indicator, i.e., a clinical variable different from the outcome for which a recognized clinical difference already exists. This approach provides a stable MID that can be used with confidence across different studies, whereas distributionbased methods, since they use statistical parameters associated with an instrument in a particular population, may not be valid in a different population [37-39].

We performed an extensive literature search to detect the smallest worthwhile effect and find an established MID for all available pain instruments related to perceived back pain in studies on nonspecific LBP (i.e., excluding specific causes such as cauda equina syndrome). We updated the search strategy adopted by Ferreira et al. in 2012 [40] who conducted a broad review of methods for determining the MID of interventions for 
LBP, which was updated up to May 2011 (Appendix 1). Using the same terms, we searched three electronic databases MEDLINE, Cumulative Index to Nursing and Allied Health Literature (CINAHL), and Embase from May 2011 to December 2015.

However, since clinical relevance depends on the instrument used and the trials used several different instruments, we had to deal with multiple pain instruments and their MIDs. Also, we found that not all the instruments have an established anchor-based MID. One option to address this problem in our case study meta-analysis [17] could have been to exclude them from the MA and pool only the RCTs that reported an instrument with an established anchor-based MID. However, because this would have limited the power of the MA and potentially introduced a selection bias, we adopted the distribution-based method proposed by Johnston [41] to obtain a sensible MID for the instruments for which an anchor-based MID was unavailable. The same approach was used for studies not reporting the pain measurement tool.

\section{Data extraction and statistical analysis}

Two authors, both experienced in systematic review methodology, independently re-extracted the data (i.e., mean difference and standard deviation) from the primary studies for each comparison and abstracted the pain measurement instrument (e.g., VAS) from the studies included in the previous Cochrane review [17]. We performed the metaanalyses in MID units and also in SMD for comparison. All effect sizes are reported with their corresponding 95\% confidence interval $(\mathrm{CI})$. The mean difference was calculated as control minus treatment in all meta-analyses, i.e., a positive MID or SMD favors MBR over usual care.

To obtain a valid MID for the instruments lacking an anchor-based one, we first calculated the standard deviation ratio (SDR) of the studies with an anchor-based MID, i.e., the anchor-based MID of the instrument divided by the baseline standard deviation for the control group or, if not reported, the standard deviation at the end-of-treatment for the same group [16]. For each study that used an instrument without an anchor-based MID, we multiplied the SD (baseline or the end-oftreatment) by the median SDR of the studies with an anchor-based MID [16].

Next, we calculated MID units as the mean difference of individual studies divided by the MID established for the $j$ instrument used in the trial and then pooled them. According to Johnstone, results expressed in MID units can be interpreted as follows: if the overall effect size is greater than 1, many patients are likely to gain clinically important benefits from the treatment; if it lies between 0.5 and 1.0, an appreciable number will benefit; and if it falls below 0.5 MID units, few may achieve important benefits [16].
The use of MID units makes it easier for clinicians to understand the effects of an intervention since they are more confident with clinical scales, such as the NRS, than with non-clinical measures such as SMD. In fact, a pooled estimate expressed in MID units can be easily converted between scales by multiplying it by the established MID of a given instrument. For example, the established MID for NRS is 2 points. By multiplying the pooled estimate in MID units by 2 points, we obtain the effect size in NRS units. All analyses were performed using R software [42].

\section{Results}

\section{Selection of the MID for each instrument}

We found 22 studies reporting an anchor-based MID for pain measurement instruments in musculoskeletal back pain (Additional file 1: Table S1): 14 used a NRS, 5 used a VAS, and one study each used the Pain Self-Efficacy Questionnaire (PSEQ), the Patient-Specific Functional Scale (PSFS), and the 11-Face Faces Pain Scale [17].

For the studies that reported an anchor-based MID for VAS and NRS, we used the minimal important change values proposed by Ostelo et al. [43] because they were expressed by a consensus expert panel and because they were consistent with the MID values retrieved in all the other studies found. Accordingly, we assumed an anchor-based MID of 15 for VAS and of 2 for NRS. The MIDs for the other instruments were not employed, since they were not used in the trials in our case study meta-analysis. We found no study that reported an anchor-based MID for the remaining instruments (i.e., SF-36 Body Pain Index) in low back pain.

\section{Descriptive characteristics}

The mean change in pain from baseline in the MBR and the usual care groups at short-, medium-, and long-term follow-up are reported in Additional file 2: Table S2. Nine studies were reported on short-term follow-up after MBR versus usual care for reduction of back pain, 6 on medium-term, and 7 on long-term (Table 1).

Overall, the three case study meta-analyses included 13 trials. Ten RCTs employed two widely used disease-specific pain instruments: the NRS and VAS. Both instruments have demonstrated their validity and responsiveness in various settings [44]. Another valid pain instrument [45] reported in two trials $[20,21]$ was the SF-36 Body Pain Index. One

\begin{tabular}{llll}
\multicolumn{3}{l}{ Table 1 Pain measurement instruments and follow-up period } \\
\hline Tool & $\begin{array}{l}\text { Short term } \\
(n=9)\end{array}$ & $\begin{array}{l}\text { Medium term } \\
(n=6)\end{array}$ & $\begin{array}{l}\text { Long term } \\
(n=7)\end{array}$ \\
\hline NRS & 2 & 2 & 3 \\
VAS & 4 & 3 & 4 \\
SF-36 Body Pain Index & 2 & 1 & 0 \\
Not reported & 1 & 0 & 0 \\
\hline
\end{tabular}


trial did not report the instrument employed [19]. We refer the reader to the published Cochrane review for details on the descriptive characteristics of the trials [17].

\section{Imputation of the MID for instruments without an anchor-based MID}

The distribution of the SDR for each instrument with an anchor-based MID is presented in Table 2. In the metaanalysis of the short-term period, the overall median SDR was 0.83 , which we used to calculate the distributionbased MIDs for the three studies that used an instrument without an established MID. The imputed MID was: 24.8 for Tavafian 2008 [21] (using SF-36 Body Pain Index, range 0-100); 19.5 for Tavafian 2011 [20] (SF-36 Body Pain Index); 2.6 for Moix 2003 [19] (no scale reported). In the medium-term comparison, the overall median SDR was 0.79. The imputed MID was 20.4 for Tavafian 2011 [20] (SF-36 Body Pain Index). All studies had an established MID in the long-term comparison.

\section{Results of meta-analyses in MID units and comparison with results from pooling SMD}

Consistent with the Cochrane review [17], after pooling the SMDs we found a statistically significant effect in favour of MBR over usual care for pain relief in all comparisons. For the meta-analyses in MID units, the pooled estimate of the effect was 0.75 MID units (95\% confidence interval [CI] 0.27-1.24; $\mathrm{I}^{2} 88.7,95 \%$ CI 72.8-97.3, Fig. 1) for the short-term comparison; 0.86 MID units (95\% CI 1.33-0.39; I I 83.7, 95\% CI 52.7-97.8, Fig. 2) for the medium-term comparison; and 0.27 MID units (95\% CI $0.07-0.48 ; \mathrm{I}^{2}$ 23.2, 95\% CI 0-79.2, Fig. 3) for the long-term comparison. Given that the anchor-based established MID for NRS is 2, this finding translates into an improvement of $1.50,1.72$, and 0.54 points, respectively, on the NRS ten points scale.

Table 2 Distribution of SDR for each instrument with an anchor-based MID

\begin{tabular}{llll}
\hline MBT vs Usual Care & Median SDR & Minimum SDR & Maximum SDR \\
\hline Short-term (studies with & established MID, $n=6 / 9)$ & \\
NRS $(n=2)$ & 1.256 & 1.053 & 1.460 \\
VAS 0-10 cm $(n=4)$ & 0.698 & 0.638 & 0.938 \\
Overall $(n=6)$ & 0.826 & 0.638 & 1.460 \\
Medium-term (studies with established MID, $n=5 / 6)$ \\
NRS ( $n=2)$ & 0.931 & 0.909 & 0.952 \\
VAS 0-10 cm ( $n=3)$ & 0.682 & 0.622 & 0.789 \\
Overall $(n=5)$ & 0.789 & 0.622 & 0.952 \\
Long-term (studies with established MID, $n=7 / 7)$ & \\
NRS ( $n=3)$ & 0.909 & 0.769 & 0.952 \\
VAS 0-10 cm $(n=4)$ & 0.732 & 0.560 & 1.071 \\
Overall $(n=7)$ & 0.769 & 0.560 & 1.071 \\
\hline
\end{tabular}

Comparison of the meta-analyses in SMD and MID units (Table 3) shows that the results are consistent across the two units of measurement: the overall estimates are statistically significant, irrespective of the approach used. From a strictly statistical point of view, we can conclude that MBR is superior to usual care at all follow-up times. All point estimates in MID units are $<1$, however, i.e., the average change from baseline to the end of follow-up is smaller than the MID (Fig. 4). Also, the pooled estimates need to be carefully interpreted in light of the substantial statistical heterogeneity using the $\mathrm{I}^{2}$ statistics in the metaanalyses of the short- and medium-term.

\section{Discussion}

We noted a limited advantage of MBR versus usual care at all follow-up times when the results of the meta-analyses were expressed in MID units. Though there was a statistically significant difference in pain relief between the two treatments, all point estimates were smaller than 1 MID, which is, by definition, the clinical sizeable benefit. Metaanalyses almost always give nominally statistically significant results at $p<0.05$ for the difference between two treatments. This is not relevant for health care professionals, however, if the effect size is not large enough to have a practical impact on patients. Reporting MID units assumes a patient-centred perspective: treatment with a MID above 1 is expected to have important benefits for the majority of patients but for very few if below 0.5 [46]. Accordingly, comparison of MBR versus usual care for short-and medium-term relief of back pain shows that there is a real but clinically modest difference (slightly lower than $1 \mathrm{MID}$ ) in favour of MBR. In the long-term comparison, the benefit, albeit statistically significant, is not clinically relevant, on average, as the pooled estimated is less than one-third of 1 MID.

A systematic review published in 2007 [47] reported no effectiveness of MBR versus usual care for pain (only one of the 7 studies reported a positive effect). In contrast, the 2014 Cochrane review that we selected as case study showed that MBR, when analyzed in SMD, significantly reduced back pain in the long-term. However, the small effect and the moderate quality of evidence rated with the GRADE system led the authors to conclude that the superiority of MBR may be clinically relevant, a conclusion also remarked on in a recent update [34]. In our study we better quantified the clinical relevance by using the MID as a benchmark for drawing conclusions. This could be particularly relevant in the context of clinical recommendations.

According to GRADE guidelines [48], when evaluating imprecision, the authors of systematic reviews should consider whether the CIs of the effect size include appreciable benefit or harm [49]. Reporting meta-analyses in MID units can help readers and stakeholders judge at 


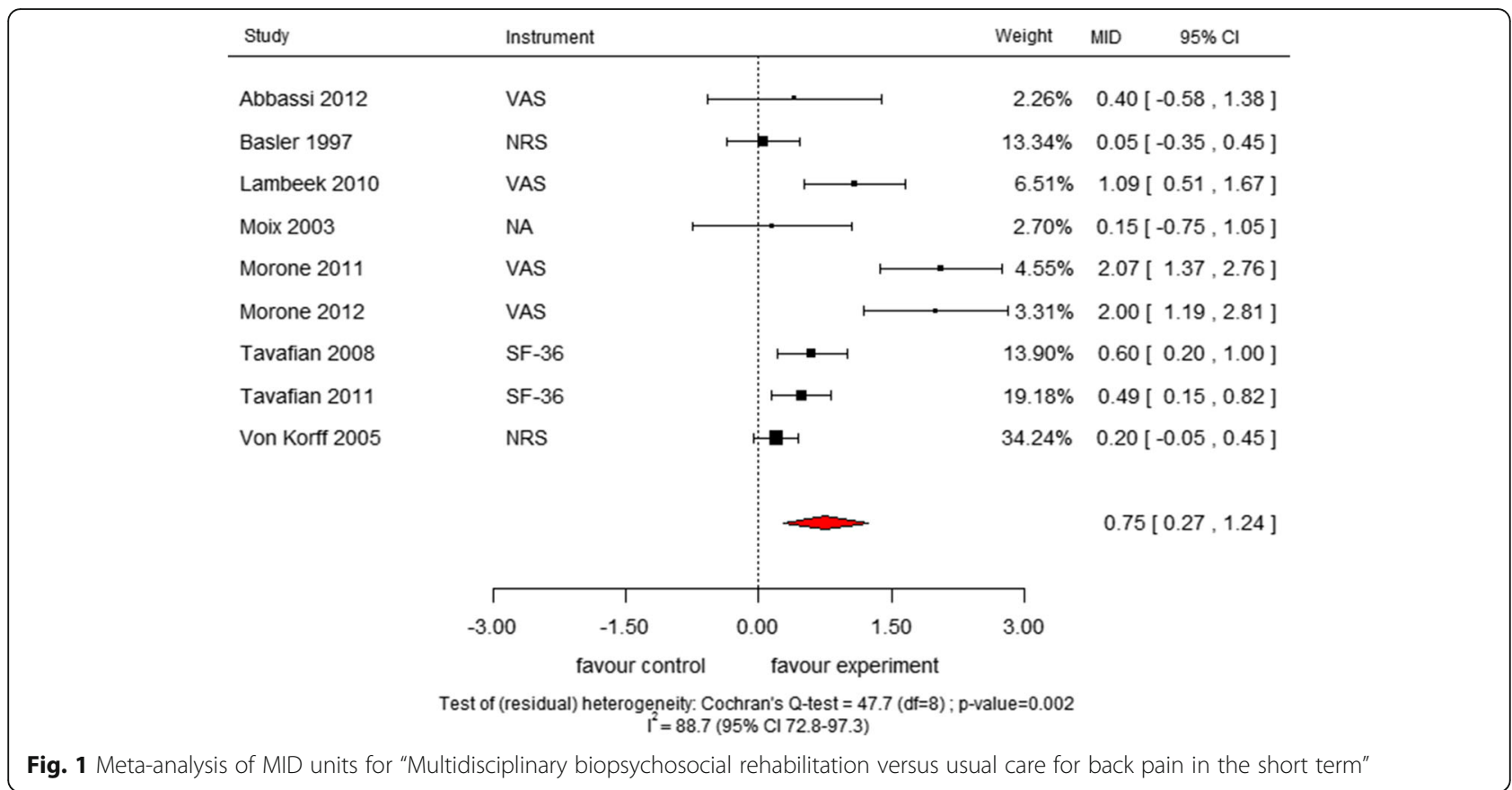

a glance the precision of the overall effect in terms of clinical relevance and the amount of benefit or harm against a clear anchor point. Furthermore, optimal information size can be directly related to both clinical and statistical significance. Drawing conclusions from metaanalyses based only on statistical significance may be misleading, however, especially if associated with a high prevalence of small studies and poor reporting, as is typical of the rehabilitation literature $[10,50]$. There is a need to move beyond the $p$-value cliché and to focus on the magnitude of benefit since interventions of limited value sap valuable time and resources from other interventions that might have more substantial effects.

The goal of MBR is to teach individuals to cope with their pain. In doing so, the aim is to modify deeplyrooted attitudes and beliefs, as they may contribute to prolonging back pain by activating physical and emotional "triggers" [51]. It is expected that interventions will produce clinical relevant benefits in both the shortand long-term. We found no evidence of clinically

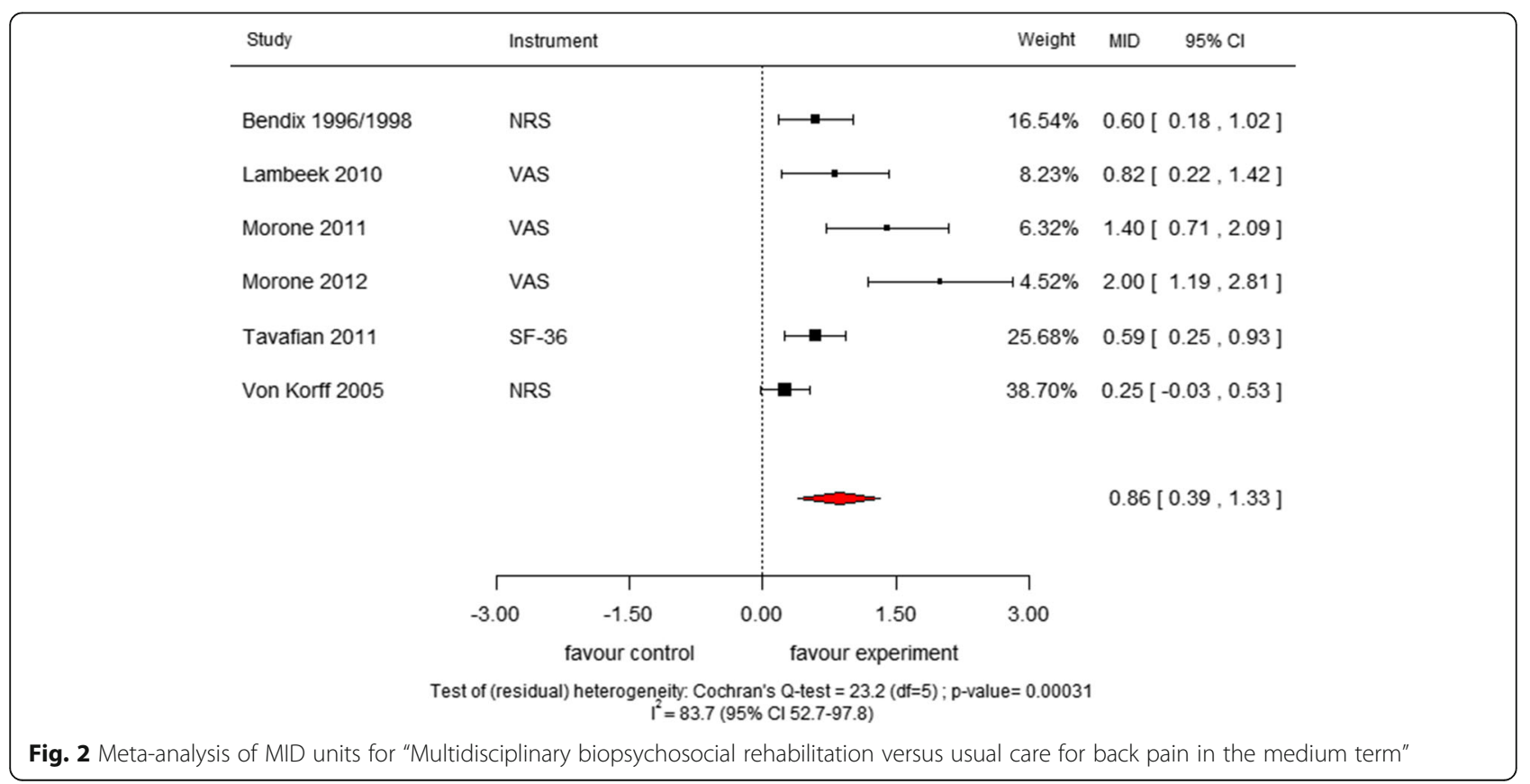




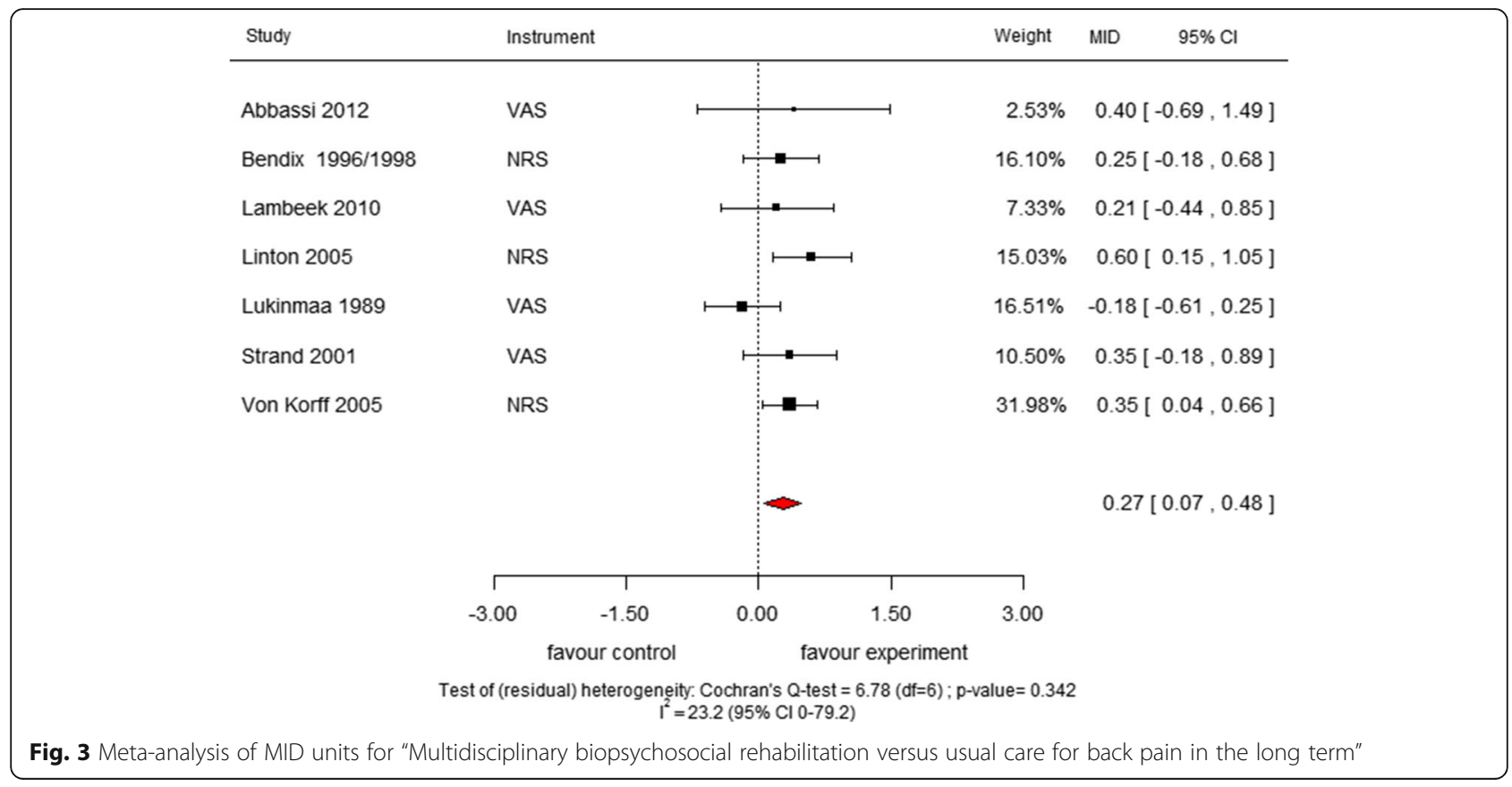

relevant long-term results. One possible explication is that, after the acute phase, when the specialist sees the patient, measures baseline pain, and starts the therapy, pain may decrease over time regardless of treatment. This makes potential long-term effects smaller and more difficult to detect [52].

We showed that, in a clinically meaningful summary estimate such as the MID, the results of a meta-analysis can be interpreted differently by clinicians and patients. The main finding of our study has possible implications for recommending MBR. Multidisciplinary biopsychological rehabilitation is endorsed in the ACP/APS [53] in the National Disease Management [54] and the 2016 NICE draft guidelines [55]. Based on our results, and given the potentially high cost of MBR, these indications need to be re-considered.

The MID unit approach has some limitations in particular instances. First, the use of MID units requires that previous studies have reported an estimate of the MID (possibly an anchor-based MID) from several trials. Currently, few instruments that assess an outcome have an established MID: one study including a large cohort of trials $(n=185)$ on LBP rehabilitation found 70 different pain measurement instruments [10]. In contrast, we

Table 3 Meta-analyses in SMD and MID units for each follow-up period

\begin{tabular}{lll}
\hline MBR vs. Usual Care & SMD $(95 \% \mathrm{Cl})$ & MID units $(95 \% \mathrm{Cl})$ \\
\hline Short-term & $0.56(0.28-0.83)$ & $0.75(0.27-1.24)$ \\
Medium-term & $0.60(0.34-0.87)$ & $0.86(0.39-1.33)$ \\
Long-term & $0.21(0.04-0.27)$ & $0.27(0.07-0.48)$ \\
\hline
\end{tabular}

found only 5 instruments that had an anchor-based MID. Second, the MID is informative only about the comparison of a treatment versus a control (i.e., usual care or placebo). If we compare two different treatments, the MID value needs to be modified to account for the effect of the control treatment. For example, in the comparison of MBR versus pharmacological treatment, we should not apply the same MID that we used against usual care because the latter will already have a sizeable effect on pain relief and a smaller additional increase could be interpreted as clinically relevant. In addition, in these meta-analyses, and regardless of the unit of analysis, usual care is not the same for all studies. Also, we used a MID that does not distinguish between chronic and acute pain [43]. Finally, meta-analyses reported in MID units are vulnerable to unexperienced, oversimplified interpretation unless we keep in mind that when we define a MID, we choose a single value while, in reality, the MID is subjective, i.e., the clinical relevance of a change in outcome may be perceived differently from patient to patient.

\section{Conclusions}

Chronic low back pain carries a poor prognosis. In such patients, a multidisciplinary rehabilitation program is believed to improve long-term pain. Performing and reporting meta-analyses in MID units proved to be useful for enhancing clinical interpretability of the results. By applying this method we were able to show a difference in the interpretation and conclusion of metaanalysis reported in SMD units: MBR has a clinically modest advantage over usual care only in the short-and 


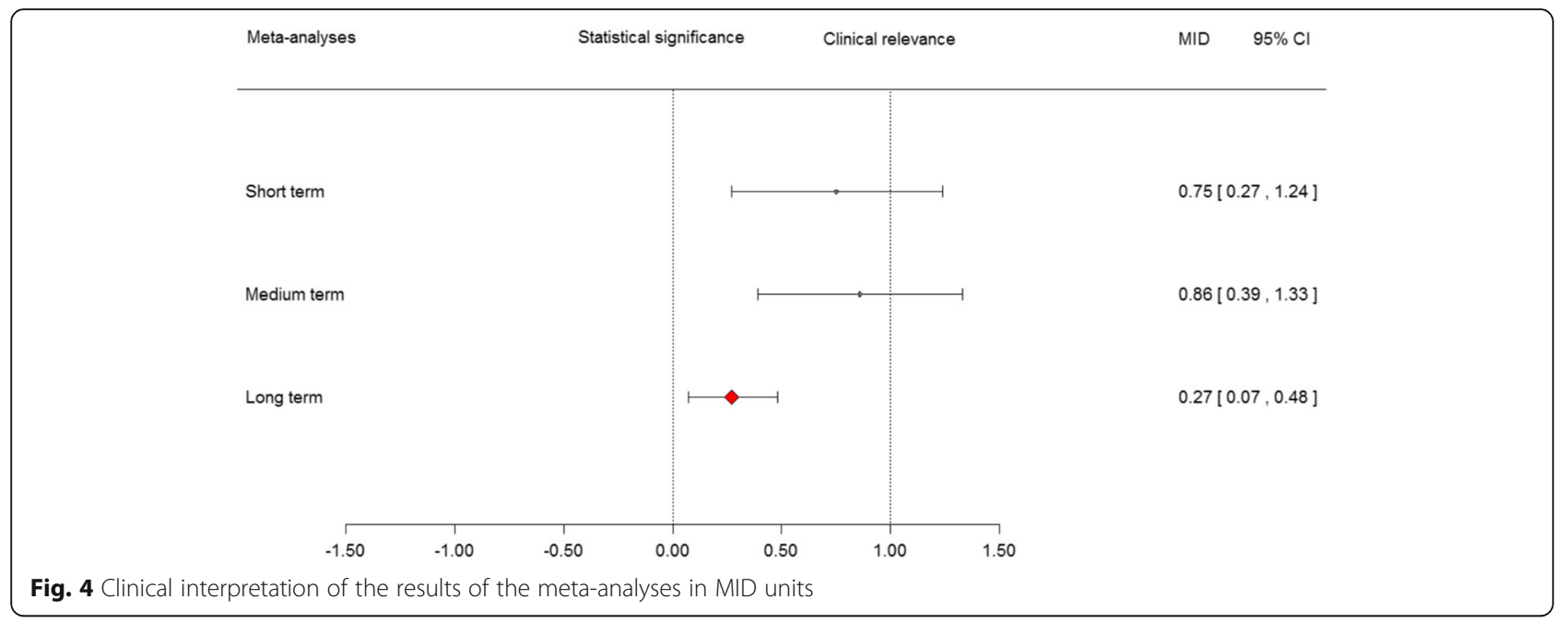

medium-term follow-up. Its advantage over usual care, although statistically significant, is not clinically relevant in the long-term.

\section{Appendix 1}

MEDLINE search strategy for established MIDs.

(clinical importan* OR worthwhile change* OR important difference OR minimal clinically significant OR clinically important difference OR clinically significant difference OR clinical significance OR clinical effect* OR treatment effect* OR minimal differen* OR minim* clinically important difference OR responsive* OR "smallest detectable change" OR "minimal detectable change" OR "minimal important change" OR minim*clinical" important change OR minim* important difference OR patient expectation OR patient preference OR clinical relevance OR patient perspective OR minim* change* OR sensitivity to change OR standardized response mean) AND ("low back pain" OR "back pain" OR "spinal pain")

\section{Additional files}

Additional file 1: Table S1. Anchor-based MID for pain measurement instruments in musculoskeletal back pain. (DOCX 49 kb)

Additional file 2: Table S2. Mean change in pain from baseline in the MBR and the usual care groups. (DOCX $40 \mathrm{~kb}$ )

\section{Abbreviations}

Cl: Confidence intervals; LBP: Low back pain; MA: Meta-analysis; MBR: Multidisciplinary biopsychosocial rehabilitation; MID: Minimal important difference; NRS: Numerical rating scale; RCT: Randomized controlled trials; SD: Standard deviation; SDR: Standard deviation ratio; SMD: Standardized mean difference; VAS: Visual analogue scale

\section{Funding}

The work was supported by the Italian Ministry of Health (L3017) and by Italian Ministry of Health (Giovani Ricercatori GR-2011-02348048). The funding sources had no controlling role in the study design, data collection, analysis, interpretation, or report writing.

\section{Availability of data and materials}

All data generated or analysed during this study are included in this published article [and its Additional files].

\section{Authors' contributions}

SG, LM conceived the original idea for the research. SG, AA conducted the design study and planned the methods. SG, GC extracted data. SG, AA analysed the data and prepared the results. All authors commented on results. SG, AA drafted the manuscript. All the authors had access to the data and analysis, revised manuscript for intellectual content and approved the final version. LM, MGV are joint guarantors for this study.

Ethics approval and consent to participate Not applicable.

\section{Competing interests}

The authors declare that they have no competing interests.

\section{Publisher's Note}

Springer Nature remains neutral with regard to jurisdictional claims in published maps and institutional affiliations.

\section{Author details}

${ }^{1}$ Clinical Epidemiology Unit, I.R.C.C.S. Orthopedic Institute Galeazzi, via R Galeazzi 4, Milan, Italy. ${ }^{2}$ Center of Biostatistics for Clinical Epidemiology, School of Medicine and Surgery, University of Milano-Bicocca, Monza, Italy. ${ }^{3}$ Department of Biomedical Sciences for Health, University of Milan, Milan, Italy.

Received: 28 July 2017 Accepted: 6 May 2018

Published online: 15 May 2018

\section{References}

1. Hoy D, Bain C, Williams G, March L, Brooks P, Blyth F, Woolf A, Vos T, Buchbinder R. A systematic review of the global prevalence of low back pain. Arthritis Rheum. 2012;64:2028-37.

2. van Tulder MW, Koes BW, Bouter LM. A cost-of-illness study of back pain in the Netherlands. Pain. 1995;62:233-40.

3. Costa Lda C, Maher CG, McAuley JH, Hancock MJ, Herbert RD, Refshauge KM, Henschke N. Prognosis for patients with chronic low back pain: inception cohort study. BMJ. 2009:339:b3829.

4. Koes BW, van Tulder M, Lin CW, Macedo LG, McAuley J, Maher C. An updated overview of clinical guidelines for the management of non-specific low back pain in primary care. Eur Spine J. 2010;19:2075-94.

5. Maher C, Underwood M, Buchbinder R. Non-specific low back pain. Lancet. 2016;

6. Lee J, Gupta S, Price C, Baranowski AP. Low back and radicular pain: a pathway for care developed by the British pain society. Br J Anaesth. 2013;111:112-20. 
7. Waddell G. The back pain revolution, vol. 1. 2nd ed. London: Churchill Livingston; 2004

8. Baliki MN, Apkarian AV. Nociception, pain, negative moods, and behavior selection. Neuron. 2015;87:474-91.

9. Pergolizzi JV Jr, Raffa RB, Fleischer C, Zampogna G, Taylor R Jr. Management of moderate to severe chronic low back pain with buprenorphine buccal film using novel bioerodible mucoadhesive technology. J Pain Res. 2016;9:909-16.

10. Gianola S, Frigerio P, Agostini M, Bolotta R, Castellini G, Corbetta D, Gasparini M, Gozzer P, Guariento E, Li LC, Pecoraro V, Sirtori V, Turolla A, Andreano A, Moja L. Completeness of Outcomes Description Reported in Low Back Pain Rehabilitation Interventions: A Survey of 185 Randomized Trials. Physiother Can. 2016;68(3):267-74.

11. Chapman JR, Norvell DC, Hermsmeyer JT, Bransford RJ, DeVine J, McGirt MJ, Lee MJ. Evaluating common outcomes for measuring treatment success for chronic low back pain. Spine (Phila Pa 1976). 2011;36:S54-68.

12. Deyo RA, Battie M, Beurskens AJ, Bombardier C, Croft P, Koes B, Malmivaara A Roland M, Von Korff M, Waddell G. Outcome measures for low back pain research. A proposal for standardized use. Spine (Phila Pa 1976). 1998;23:2003-13.

13. Deeks JJ, Higgins JPT, Altman DG (editors). Chapter 9: Analysing data and undertaking meta-analyses. In: Higgins JPT, Green S (editors). Cochrane Handbook for Systematic Reviews of Interventions. Chichester: Wiley; 2008.

14. Schunemann HJ, Oxman AD, Vist GE, Higgins JPT, Deeks JJ, Glasziou P, Guyatt GH. Chapter 12: Interpreting results and drawing conclusions. In: Higgins JPT, Green S (editors), Cochrane Handbook for Systematic Reviews of Interventions. Chichester: Wiley; 2008.

15. Schunemann $H J$, Puhan $M$, Goldstein $R$, Jaeschke $R$, Guyatt $G H$. Measurement properties and interpretability of the chronic respiratory disease questionnaire (CRQ). COPD. 2005;2:81-9.

16. Johnston BC, Thorlund K, Schunemann HJ, Xie F, Murad MH, Montori VM, Guyatt GH. Improving the interpretation of quality of life evidence in metaanalyses: the application of minimal important difference units. Health Qual Life Outcomes. 2010;8:116.

17. Kamper SJ, Apeldoorn AT, Chiarotto A, Smeets RJ, Ostelo RW, Guzman J, van Tulder MW. Multidisciplinary biopsychosocial rehabilitation for chronic low back pain. Cochrane Database Syst Rev. 2014;9:CD000963.

18. Cates CJ, Stovold E, Emma JW. How to make sense of a Cochrane systematic review. Breathe. 2014;10:134-44.

19. Moix J, Canellas M, Osorio C, Bel X, Givent F, Martos A. Efficacy of an interdisciplinary educational program in patients with chronic back pain. Dolor. 2003;18:149-57.

20. Tavafian SS, Jamshidi AR, Mohammad K. Treatment of chronic low back pain: a randomized clinical trial comparing multidisciplinary group-based rehabilitation program and oral drug treatment with oral drug treatment alone. Clin J Pain. 2011;27:811-8.

21. Tavafian SS, Jamshidi AR, Montazeri A. A randomized study of back school in women with chronic low back pain: quality of life at three, six, and twelve months follow-up. Spine (Phila Pa 1976). 2008;33:1617-21.

22. Morone G, losa M, Paolucci T, Fusco A, Alcuri R, Spadini E, Saraceni VM, Paolucci S. Efficacy of perceptive rehabilitation in the treatment of chronic nonspecific low back pain through a new tool: a randomized clinical study. Clin Rehabil. 2012;26:339-50.

23. Morone G, Paolucci T, Alcuri MR, Vulpiani MC, Matano A, Bureca I, Paolucci S, Saraceni VM. Quality of life improved by multidisciplinary back school program in patients with chronic non-specific low back pain: a single blind randomized controlled trial. Eur J Phys Rehabil Med. 2011:47:533-41.

24. Abbasi M, Dehghani M, Keefe FJ, Jafari H, Behtash H, Shams J. Spouseassisted training in pain coping skills and the outcome of multidisciplinary pain management for chronic low back pain treatment: a 1-year randomized controlled trial. Eur J Pain. 2012;16:1033-43.

25. Von Korff M, Balderson BH, Saunders K, Miglioretti DL, Lin EH, Berry S, Moore $J E$, Turner JA. A trial of an activating intervention for chronic back pain in primary care and physical therapy settings. Pain. 2005;113:323-30.

26. Linton SJ, Boersma K, Jansson M, Svard L, Botvalde M. The effects of cognitive-behavioral and physical therapy preventive interventions on painrelated sick leave: a randomized controlled trial. Clin J Pain. 2005;21:109-19.

27. Basler HD, Jakle C, Kroner-Herwig B. Incorporation of cognitive-behavioral treatment into the medical care of chronic low back patients: a controlled randomized study in German pain treatment centers. Patient Educ Couns. 1997:31:113-24
28. Strand LI, Ljunggren AE, Haldorsen EM, Espehaug B. The impact of physical function and pain on work status at 1-year follow-up in patients with back pain. Spine (Phila Pa 1976). 2001;26:800-8.

29. Bendix AE, Bendix T, Haestrup C, Busch E. A prospective, randomized 5-year follow-up study of functional restoration in chronic low back pain patients. Eur Spine J. 1998;7:111-9.

30. Bendix AF, Bendix T, Vaegter K, Lund C, Frolund L, Holm L. Multidisciplinary intensive treatment for chronic low back pain: a randomized, prospective study. Cleve Clin J Med. 1996;63:62-9.

31. Lambeek LC, Bosmans JE, Van Royen BJ, Van Tulder MW, Van Mechelen W, Anema JR. Effect of integrated care for sick listed patients with chronic low back pain: economic evaluation alongside a randomised controlled trial. BMJ. 2010;341:C6414.

32. Lambeek LC, van Mechelen W, Knol DL, Loisel P, Anema JR. Randomised controlled trial of integrated care to reduce disability from chronic low back pain in working and private life. BMJ. 2010;340:c1035.

33. Lukinmaa A. Low back pain as a biopsychosocial problem. A controlled clinical trial and a costeffectiveness analysis[Finnish]. Kansanelakelaitoksen Julkaisuja. 1989:ML:1-90.

34. Kamper SJ, Apeldoorn AT, Chiarotto A, Smeets RJ, Ostelo RW, Guzman J, van Tulder MW. Multidisciplinary biopsychosocial rehabilitation for chronic low back pain: Cochrane systematic review and meta-analysis. BMJ. 2015;350:h444.

35. Ostelo RW, de Vet HC. Clinically important outcomes in low back pain. Best Pract Res Clin Rheumatol. 2005;19:593-607.

36. van der Roer N, Ostelo RW, Bekkering GE, van Tulder MW, de Vet HC. Minimal clinically important change for pain intensity, functional status, and general health status in patients with nonspecific low back pain. Spine (Phila Pa 1976). 2006;31:578-82.

37. Terwee CB, Roorda LD, Dekker J, Bierma-Zeinstra SM, Peat G, Jordan KP, Croft P, de Vet HC. Mind the MIC: large variation among populations and methods. J Clin Epidemiol. 2010;63:524-34.

38. Turner $D$, Schunemann $H J$, Griffith $L E$, Beaton $D E$, Griffiths AM, Critch JN, Guyatt GH. The minimal detectable change cannot reliably replace the minimal important difference. J Clin Epidemiol. 2010;63:28-36.

39. Crosby RD, Kolotkin RL, Williams GR. Defining clinically meaningful change in health-related quality of life. J Clin Epidemiol. 2003;56:395-407.

40. Ferreira ML, Herbert RD, Ferreira PH, Latimer J, Ostelo RW, Nascimento DP, Smeets RJ. A critical review of methods used to determine the smallest worthwhile effect of interventions for low back pain. J Clin Epidemiol. 2012; 65:253-61.

41. Johnston BC, Thorlund K, da Costa BR, Furukawa TA, Guyatt GH. New methods can extend the use of minimal important difference units in meta-analyses of continuous outcome measures. J Clin Epidemiol. 2012;65:817-26.

42. R Core Team. R: A language and environment for statistical computing. Vienna: R Foundation for Statistical Computing. ISBN 3-900051-07-0, 2014. http://www.R-project.org/.

43. Ostelo RW, Deyo RA, Stratford P, Waddell G, Croft P, Von Korff M, Bouter LM, de Vet HC. Interpreting change scores for pain and functional status in low back pain: towards international consensus regarding minimal important change. Spine (Phila Pa 1976). 2008;33:90-4.

44. Good M, Stiller C, Zauszniewski JA, Anderson GC, Stanton-Hicks M, Grass JA. Sensation and distress of pain scales: reliability, validity, and sensitivity. J Nurs Meas. 2001;9:219-38.

45. Ware JE Jr, Gandek B. Overview of the SF-36 health survey and the international quality of life assessment (IQOLA) project. J Clin Epidemiol. 1998;51:903-12.

46. Guyatt GH, Juniper EF, Walter SD, Griffith LE, Goldstein RS. Interpreting treatment effects in randomised trials. BMJ. 1998:316:690-3.

47. van Geen JW, Edelaar MJ, Janssen M, van Eijk JT. The long-term effect of multidisciplinary back training: a systematic review. Spine (Phila Pa 1976). 2007;32:249-55.

48. Guyatt GH, Oxman AD, Schunemann HJ, Tugwell P, Knottnerus A. GRADE guidelines: a new series of articles in the journal of clinical epidemiology. J Clin Epidemiol. 2011;64:380-2.

49. Guyatt GH, Oxman AD, Kunz R, Brozek J, Alonso-Coello P, Rind D, Devereaux PJ, Montori VM, Freyschuss B, Vist G, et al. GRADE guidelines 6. Rating the quality of evidence-imprecision. J Clin Epidemiol. 2011;64:1283-93.

50. Gianola S, Gasparini M, Agostini M, Castellini G, Corbetta D, Gozzer P, Li LC, Sirtori V, Taricco M, Tetzlaff JM, et al. Survey of the reporting characteristics of systematic reviews in rehabilitation. Phys Ther. 2013;93:1456-66. 
51. Beattie PF, Silfies SP. Improving long-term outcomes for chronic low back pain: time for a new paradigm? J Orthop Sports Phys Ther. 2015;45:236-9.

52. Whitney CW, Von Korff M. Regression to the mean in treated versus untreated chronic pain. Pain. 1992:50:281-5.

53. Chou R, Qaseem A, Snow V, Casey D, Cross JT, Shekelle P, Owens DK. Diagnosis and treatment of low back pain: a joint clinical practice guideline from the American college of physicians and the American pain society. Ann Intern Med. 2007;147:478-91.

54. German Medical Association (BÄK); National Association of Statutory Health Insurance Physicians (KBV); Association of Scientific Medical Societies (AWMF). National Disease Management Guideline 'Low back pain' - Short Version, 1st edition. Version 5. 2011 last amended: October 2015. Available from: http://www.kreuzschmerz.versorgungsleitlinien.de; [accessed 20 October 2017 ]; https://doi.org/10.6101/AZQ/000252; Internet: http://www. versorgungsleitlinien.de, http://www.awmf-leitlinien.de.

55. de Rooij M, van der Leeden M, Avezaat E, Hakkinen A, Klaver R, Maas T, Peter WF, Roorda LD, Lems WF, Dekker J. Development of comorbidityadapted exercise protocols for patients with knee osteoarthritis. Clin Interv Aging. 2014;9:829-42.

Ready to submit your research? Choose BMC and benefit from:

- fast, convenient online submission

- thorough peer review by experienced researchers in your field

- rapid publication on acceptance

- support for research data, including large and complex data types

- gold Open Access which fosters wider collaboration and increased citations

- maximum visibility for your research: over $100 \mathrm{M}$ website views per year

At BMC, research is always in progress.

Learn more biomedcentral.com/submissions 